\title{
Evaluation of routine basal serum calcitonin measurement for an early diagnosis of medullary thyroid carcinoma.
}

Authors

Dr Entela Puca1; Dr Kadir Burak Koza1; Dr Emiljana Huti1; Dr Sonila Bitri41; Dr Albana Daka1; Dr Edmond Puca2; Prof Dr Agron Ylli2

Hospital

Objectives:

Medullary thyroid carcinoma (MTC) is characterized by a high concentration of serum calcitonin. Routine measurement of serum calcitonin concentration has been advocated for detection of MTC among patients with nodular thyroid diseases. The aims of our study were to identify medullary thyroid cancer (MTC) in its earliest stages by screening patients with basal calcitonin measurements and to determine whether basal serum calcitonin measurements should be a part of our routine evaluation of a nodular goiter.

\section{Methods:}

\section{Results:}

We performed routine measurement of basal serum calcitonin levels from 151 patients( male 30 and female 121 with nodular goiter. The average age was 47 years old (range 19-76).

Patients with FNAC or ultrasound suspicious of any kind of thyroid carcinoma and patients with elevated basa calcitonin level underwent surgery.

Three patients ( $1.9 \%$ ) had elevated basal serum calcitonin levels ranging between $129-7493 \mathrm{pg} / \mathrm{mL}$. FNAC was suggestive of MTC in 2 from 3 patient with elevated calcitonin. MTC was confirmed in all patients, with elevated calcitonin. Differentiated thyroid carcinoma, mostly papillary, was confirmed at histology in 40 subjects. The prevalence of MTC, diagnosed by serum CT measurement among 136 patients with nodular thyroid disease was high: $1.9 \%$ of all thyroid nodules and $7 \%$ of all thyroid carcinomas. Serum CT measurement was superior to FNAC in suggesting the diagnosis of MT

Conclusions:

calcitonin measurement is an effective method for the diagnosis of MTC .It is superior to FNAB for diagnosis of MTC. High basal serum calcitonin levels increase the chance of curative therapy by diagnosing it in the early stages. 